\title{
Classification of tumor microenvironment immune types based on immune response-associated gene expression
}

\author{
RYOTA KONDOU $^{1}$, AKIRA IIZUKA ${ }^{1}$, CHIZU NONOMURA $^{1}$, HARUO MIYATA ${ }^{1}$, TADASHI ASHIZAWA ${ }^{1}$, \\ TAKESHI NAGASHIMA ${ }^{2,3}$, KEIICHI OHSHIMA ${ }^{4}$, KENICHI URAKAMI ${ }^{3}$, MASATOSHI KUSUHARA ${ }^{5}$, \\ $\mathrm{KEN} \mathrm{YAMAGUCHI}^{6}$ and YASUTO AKIYAMA ${ }^{1}$ \\ ${ }^{1}$ Immunotherapy Division, Shizuoka Cancer Center Research Institute, Shizuoka 411-8777; ${ }^{2}$ SRL Inc., Tokyo 191-0002; \\ ${ }^{3}$ Cancer Diagnostic Research Division, ${ }^{4}$ Medical Genetics Division, and ${ }^{5}$ Regional Resources Division, \\ Shizuoka Cancer Center Research Institute; ${ }^{6}$ Office of the President, \\ Shizuoka Cancer Center Hospital, Shizuoka 411-8777, Japan
}

Received July 23, 2018; Accepted September 27, 2018

DOI: 10.3892/ijo.2018.4617

\begin{abstract}
In 2014, the Shizuoka Cancer Center launched project High-tech Omics-based Patient Evaluation (HOPE), which features whole exome sequencing (WES) and gene expression profiling (GEP) of fresh surgical specimens from cancer patients. With the development of clinical trials of programmed death-1 (PD-1)/PD-ligand 1 (PD-L1) blockade, PD-L1 expression and a high tumor mutation burden become possible biomarkers that could be used to predict immune responses. In this study, based on WES and GEP data from 1,734 tumors from the HOPE project, we established a tumor microenvironment (TME) immune-type classification consisting of 4 types to evaluate the immunological status of cancer patients and analyze immunological pathways specific for immune types. Project HOPE was conducted in accordance with the Ethical Guidelines for Human Genome and Genetic Analysis Research with the approval of the Institutional Review Board. Based on the expression level of the PD-L1 and $\mathrm{CD} 8 \mathrm{~B}$ genes, the immunological status was divided into 4 types as follows: A, PD- $\mathrm{L}^{+}{ }^{+} \mathrm{CD}^{2} \mathrm{~B}^{+}$; $\mathrm{B}, \mathrm{PD}-\mathrm{L1}^{+} \mathrm{CD} 8 \mathrm{~B}$; C, PD-L1 ${ }^{-C D} 8 \mathrm{~B}^{-}$; and $\mathrm{D}$, PD-L1 ${ }^{-} \mathrm{CD} 8 \mathrm{~B}^{+}$. Type A, with $\mathrm{PD}-\mathrm{L}^{+}$and $\mathrm{CD} \mathrm{B}^{+}$, exhibited an upregulation of cytotoxic T lymphocyte (CTL) killing-associated genes, T-cell activation
\end{abstract}

Correspondence to: Dr Yasuto Akiyama, Immunotherapy Division, Shizuoka Cancer Center Research Institute, 1007 Shimonagakubo, Nagaizumi-cho, Sunto-gun, Shizuoka 411-8777, Japan

E-mail: y.akiyama@scchr.jp

Abbreviations: PD-1, programmed death-1; PD-L1, programmed death-ligand 1; MSI, microsatellite instability; CTL, cytotoxic T lymphocyte; DC, dendritic cell; WES, whole-exome sequencing; GEP, gene expression profiling; SNV, single nucleotide variant

Key words: whole exome sequencing, gene expression profiling, tumor microenvironment, programmed death-1/programmed death-ligand 1 blockade, immune response-associated genes genes, antigen-presentation and dendritic cell (DC) maturation genes, and T-cell-attracting chemokine genes, which promoted Th1 antitumor responses. By contrast, type C, with PD-L1' and CD8B-, exhibited a low expression of T-cell-activating genes and an upregulation of cancer driver gene signaling, which suggested an immune-suppressive status. With regard to hypermutator tumors, $\mathrm{PD}-\mathrm{L1}^{+}$hypermutator cases exhibited a specific upregulation of the IL6 gene compared with the PD-L1 cases. On the whole, our data indicate that the classification of the TME immune types may prove to be a useful tool for evaluating the immunological status and predicting antitumor responses and prognosis.

\section{Introduction}

In recent years, a novel type of cancer immunotherapy has been developed using specific monoclonal antibodies targeting immune checkpoint molecules, such as cytotoxic T-lymphocyte-associated antigen-4 (CTLA-4) and the programmed death-1 (PD-1)/PD-ligand 1 (PD-L1). This type of therapy has been applied in a number of clinical trials (1-5) and shows promising clinical benefits even for patients with advanced-stage disease or metastatic cancer. These accomplishments have triggered a resurgence of cancer immunotherapy and have introduced a paradigm shift in cancer treatment (6).

With regard to the immune checkpoint blockade for PD-1/PD-L1 in clinical trials, a positive PD-L1 expression, a high mutation burden and a high microsatellite instability (MSI) status are considered to be possible biomarkers for clinical responder prediction and good prognosis in melanoma, non-small cell lung cancer and colon cancer patients treated with PD-1/PD-L1 inhibitors (3,7-10). Additionally, tumor-infiltrating lymphocyte (TIL) or $\mathrm{CD}^{+} \mathrm{T}$-cells as tumor microenvironment (TME) factors have become more important parameters for a good antitumor response and the prognosis of cancer patients (11-13).

As for the immunological parameters associated with TME, Rooney et al demonstrated that the cytolytic activity of the local immune infiltrate, such as perforin and granzyme was associated with MHC class I-associated neoantigens and 
mutations of antigen presentation-related genes (14). On the other hand, Ock et al classified solid tumors into specific immune types based on PD-L1 and CD8 gene expression data derived from the Cancer Genome Atlas (TCGA) database (15).

These researchers demonstrated that TCGA-derived large-scale RNA-sequencing data constitute an appropriate model that can be used to assess the TME, as the contamination of stromal cells surrounding the tumor would proportionally influence the TME gene expression profiles in an unbiased manner. In our previous gene expression profiling (GEP) studies in the project High-tech Omics-based Patient Evaluation (HOPE), which began in 2014 at Shizuoka Cancer Center (Shizuoka, Japan) $(16,17)$, the upregulated specific gene expression associated with TIL or tumor macrophages was verified (18) partly because all tumor specimens were obtained promptly from freshly surgically resected tumors. On the other hand, frozen tumor specimens were analyzed in the TCGA study $(14,15)$.

In the current study, we classified tumors registered in the HOPE project into 4 categories based on the expression level of the PD-L1 and CD8B genes according to Ock et al (15), and the association with the immune response-associated gene panel was investigated among 4 immune-type categories for the purpose of the appropriate evaluation of immunological status.

\section{Materials and methods}

Study design. The Shizuoka Cancer Center launched project HOPE in 2014, which is based on multi-omics analyses, including whole exome sequencing (WES) and GEP. Ethical approval for the HOPE study was obtained from the institutional review board at the Shizuoka Cancer Center (authorization no. 25-33). Written informed consent was obtained from all patients enrolled in the study. All experiments using clinical samples were carried out in accordance with the Ethical Guidelines for Human Genome and Genetic Analysis Research.

Clinical specimens. Tumor tissue samples with weights of $\geq 0.1 \mathrm{~g}$ were dissected from surgical specimens, along with samples of surrounding normal tissue. The areas from which tumor samples were dissected were visually assessed as containing $\geq 50 \%$ tumor content.

RNA isolation and GEP analysis. The method of RNA isolation was described previously (17). Briefly, total RNA was extracted using the miRNeasy Mini kit (Qiagen, Hilden, Germany) according to the manufacturer's instructions. RNA samples were quantified using a NanoDrop spectrophotometer (Thermo Fisher Scientific, Waltham, MA, USA) and their quality was assessed using an Agilent 2100 Bioanalyzer (Agilent Technologies, Santa Clara, CA, USA). RNA samples with an RNA integrity number $\geq 6.0$ were used for microarray analysis. Labeled samples were hybridized to the SurePrint G3 Human Gene Expression 8x60 K v2 Microarray (Agilent Technologies). Hybridization signals were detected using a DNA Microarray Scanner (Agilent Technologies). Microarray analysis was performed in accordance with the MIAME guidelines (19).
DNA isolation and WES analysis. The method of DNA isolation was described previously (17). Briefly, DNA was extracted from tissue and blood samples using a QIAamp kit (Qiagen) and subjected to WES on the Ion Proton System (Thermo Fisher Scientific). For data analysis, single-nucleotide variants (SNVs) with quality scores $<30$ or depth of coverage $<20$ were discarded. Somatic mutations were identified by comparing the data from tumor and corresponding blood samples. Driver mutations in 138 Vogelstein driver genes (20) were defined as those identified as pathogenic in the ClinVar database. SNVs of the total exonic mutations for each sequenced tumor included nonsynonymous, synonymous, and indels/frameshift mutations.

Establishment of the immune response-associated gene panel. The content of the immune response-associated gene panel has been previously described (18). In total, 174 immune response-associated genes [67 antigen-presenting cell (APC)-associated and T-cell-associated genes, 34 cytokine- and metabolism-associated genes, 48 tumor necrosis factor (TNF) and TNF receptor superfamily genes and 25 regulatory T-cell-associated genes] were selected and used for GEP analysis (data not shown).

Association of TME immune category with immune or cancer signal pathways. Cancer patients of each TME category were profiled for immune and cancer signaling pathways using immune-response-associated and Vogelstein driver gene expression data, respectively, by means of Ingenuity Pathway Analysis (IPA) software (version Summer 2017; Qiagen).

Statistical analysis. The ratio of the expression intensity between the tumor tissue and surrounding normal tissue was calculated from normalized values. A ratio of $>2.0$ was rated as positive for gene expression. The ratio of $\log _{2}$-transformed values of the PD-L1 and CD8B genes was plotted on the vertical and horizontal axis, respectively (Fig. 1B). Based on the expression levels of the PD-L1 and CD8B genes, we classified all 1,734 tumors enrolled in the HOPE project into 4 immune types as follows: type $\mathrm{A}, \mathrm{PD}^{-\mathrm{L}^{+} \mathrm{CD}^{+} \text {; }}$

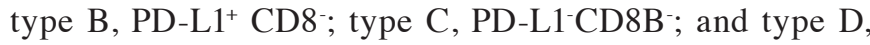
$\mathrm{PD}-\mathrm{L1}^{-} \mathrm{CD} 8 \mathrm{~B}^{+}$. The upregulated genes in expression derived from the 174 immune response-associated gene panel between TME immune type A and other types were identified using the volcano plot method. Comparing the proportion of categorical variables in each immune-type category was performed using Pearson's Chi-square test and the unpaired two-tailed t-test. Values of $\mathrm{P}<0.05$ were considered significant. Data analysis was performed using GeneSpring GX software version 13.1.1 (Agilent Technologies) and Microsoft Excel.

\section{Results}

Histological distribution and TME immune type classification of cancer patients enrolled in the HOPE project. GEP was accomplished in 1,734 pairs (without metastasis) of tumors and adjacent normal tissues derived from 17 different cancer types. Colon-rectum, lung, stomach and head and neck cancers accounted for $>60 \%$ of the total cases (Fig. 1A). The proportion of TME immune types A, B, C and D were 39.2, 26.2, 18.9 
A

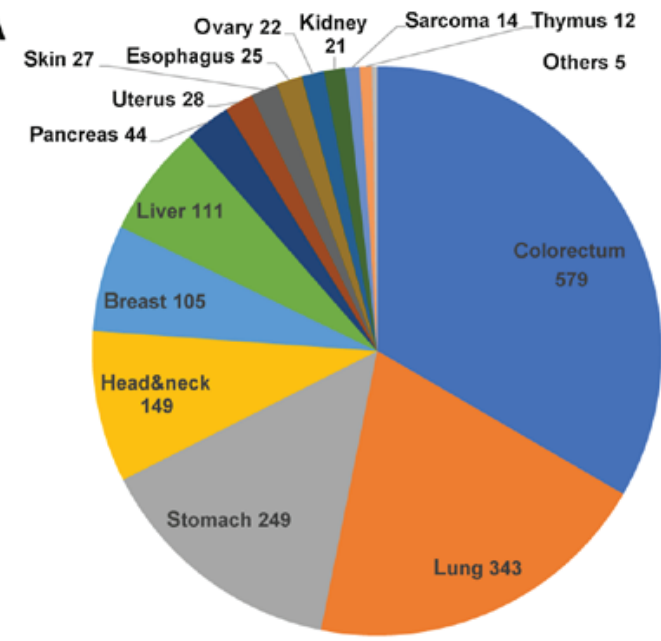

B

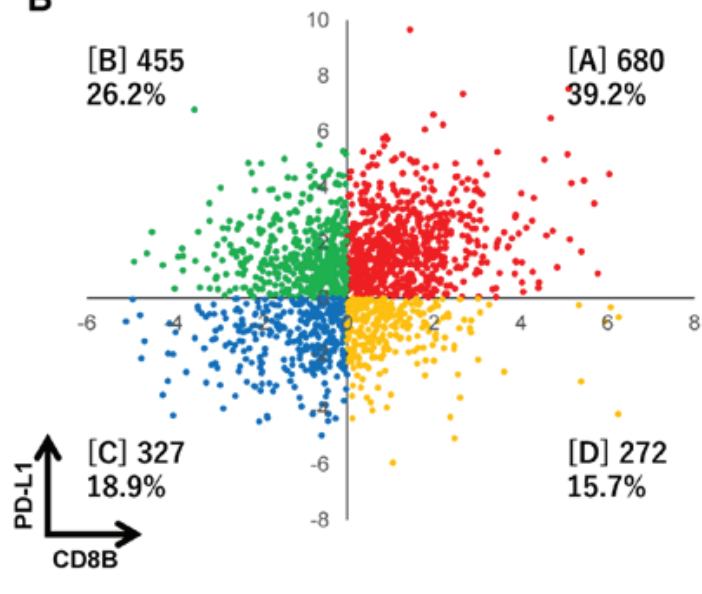

C

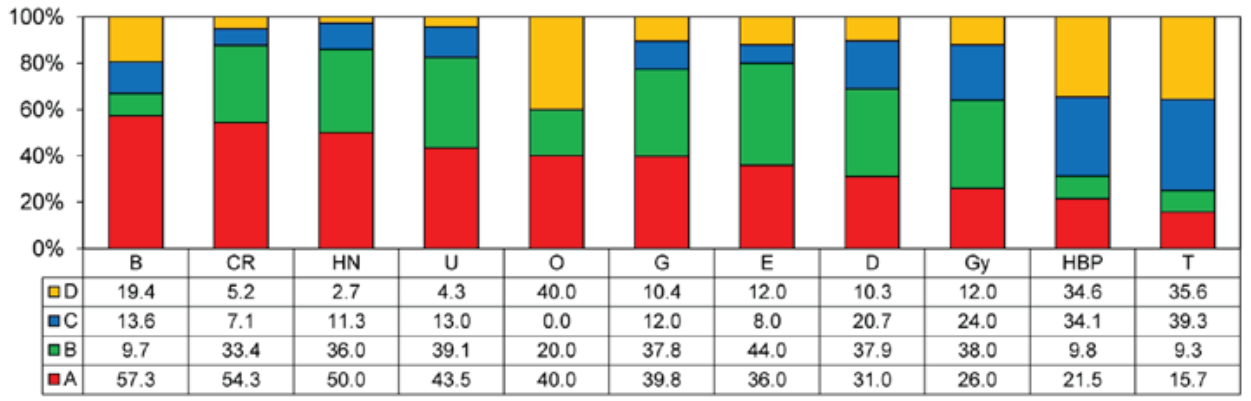

Figure 1. The classification of TME immune-type categories in various cancers registered in project HOPE. (A) The organ distribution of 1,734 tumors derived from 17 different types registered in project HOPE. (B) The proportion of TME immune types A, B, C and D based on the expression levels of both the PD-L1 and CD8B genes. Types A, B, C and D are indicated in square brackets. (C) The proportions of each immune type in various cancer types. B, breast; CR, colorectal; HN, head and neck; U, kidney; O, bone; G, stomach; E, esophagus; D, skin; Gy, uterus and ovary; HBP, liver and pancreas; and T, lung. Each value represents the proportion in percentage of each immune type. TME, tumor microenvironment; HOPE, High-tech Omics-based Patient Evaluation.

and $15.7 \%$, respectively (Fig. 1B). The proportion of type A was $>50 \%$ in breast, colon-rectum and head and neck cancers, while it was low in lung, liver and pancreatic cancers (Fig. 1C). On the other hand, the proportion of types $\mathrm{C}$ and $\mathrm{D}$ was higher in liver, pancreas and lung cancers. No clinicopathological factors, such as age, sex, smoking habit or clinical stage, was associated with immune type categories (data not shown).

Association of Vogelstein driver gene mutations and gene amplifications with TME immune types. We focused on somatic SNVs obtained by WES analysis and Vogelstein driver gene mutation profiling was investigated. The proportion of all Vogelstein driver gene mutations and TP53, KRAS, EGFR, PIK3CA, BRAF mutations were calculated in each TME immune type. The frequency of Vogelstein driver gene mutation had a tendency to be higher in types $\mathrm{C}$ and D (Fig. 2A and Table I), and so did the frequency of EGFR mutations. On the other hand, the frequency of TP53 and KRAS mutations was higher in type A compared with the other types. Moreover, all 64 gene amplifications, which have been reported previously as a gene list with a $>5$-fold change in expression and $>6$ copy numbers (17), had no significant effect on the TME immune types. However, EGFR amplification exhibited a tendency to be higher in type $\mathrm{C}$, similar to the EGFR mutation frequency (Fig. 2B and Table I).

Association of immunological cell surface markers with TME immune types. The frequencies of exhausted T-cells
$\left(\mathrm{PD}-1^{+} \mathrm{TIM}^{+}\right)$, activated effector T-cells (FAS ligand $\left.{ }^{+} \mathrm{CD} 69^{+}\right)$ and mature dendritic cells (DCs; CD11c $\left.{ }^{+} \mathrm{CD} 83^{+} \mathrm{HLA}^{-D R^{+}}\right)$ were significantly higher in types A (Fig. 3 and Table I). Additionally, the frequencies of activated natural killer (NK) cells $\left(\mathrm{CD} 16^{+} \mathrm{NCR}^{+}\right)$and macrophages $\left(\mathrm{CSF} 1 \mathrm{R}^{+} \mathrm{MSR} 1^{+}\right)$ exhibited a tendency to be higher in type A (Fig. 3 and Table I).

Association of cytolysis and helper T-cell-related cytokines with TME immune types. The frequencies of tumors positive for immuno-activation cytokine (IFNG, TNFA and IL12), immuno-suppressive cytokine (VEGFA, TGFB1, IL6 and IL10) and cytolytic factor (GZMB and PRF1) gene expressions were high in types A (Fig. 4 and Table I). In particular, the IFNG and GZMB gene expression rate was $>50 \%$ in type A.

The identification of upregulated immune response-associated genes in TME immune type A compared with other types. A total of 46 upregulated immune response-associated genes with a $>2$-fold change in expression were identified using the volcano plot (Fig. 5). These genes belonged to Th1 pathway-activating gene population, such as T-cell effector activation (FASLIG, CD40LIG, TNFSF4, TNFSF9 and TNFSF14), CTL killing (GZMB and PRF1), T-cell attraction (CCL20, CXCR6, CCL4 and CCL5), DC maturation and Th1 cytokines (IFNG, IL12, TNFA and STAT4) (Fig. 6).

Association of TME immune types with immune or cancer signaling pathways. Based on the expression data of a panel 
Table I. Comparison of immunological and genetic features between TME type A and others.

\begin{tabular}{|c|c|c|c|}
\hline Group & $\begin{array}{l}\text { Type A } \\
n=706\end{array}$ & $\begin{array}{l}\text { Others (type B, C, D) } \\
n=1,138\end{array}$ & P-value \\
\hline \multicolumn{4}{|l|}{ Genetic mutations } \\
\hline Vogelstein & $256(36.3 \%)$ & $441(38.8 \%)$ & $\mathrm{P}=0.174$ \\
\hline TP53 & $314(44.5 \%)$ & $438(38.5 \%)$ & $\mathrm{P}<0.01$ \\
\hline KRAS & $158(22.4 \%)$ & $174(15.3 \%)$ & $\mathrm{P}<0.001$ \\
\hline EGFR & $11(1.6 \%)$ & $96(8.4 \%)$ & $\mathrm{P}<0.001$ \\
\hline PIK3CA & $71(10.1 \%)$ & $106(9.3 \%)$ & $\mathrm{P}=0.498$ \\
\hline BRAF & $26(3.7 \%)$ & $39(3.4 \%)$ & $\mathrm{P}=0.709$ \\
\hline SNV no. ${ }^{a}$ & 157.8 & 110.3 & $\mathrm{P}=0.112$ \\
\hline \multicolumn{4}{|l|}{ Gene amplification ${ }^{\mathrm{b}}$} \\
\hline All 64 genes & $33(6.0 \%)$ & $64(7.4 \%)$ & $\mathrm{P}=0.178$ \\
\hline EGFR & $10(1.8 \%)$ & $17(2.0 \%)$ & $\mathrm{P}=0.096$ \\
\hline HER2 & $10(1.8 \%)$ & $14(1.6 \%)$ & $\mathrm{P}=0.858$ \\
\hline \multicolumn{4}{|l|}{ Immune cell subpopulation } \\
\hline PD- $1^{+} \mathrm{TIM}^{+}$ & $206(30.3 \%)$ & $26(2.5 \%)$ & $\mathrm{P}<0.001$ \\
\hline FAS ligand $^{+} \mathrm{CD} 69^{+}$ & $155(22.8 \%)$ & $20(1.9 \%)$ & $\mathrm{P}<0.001$ \\
\hline 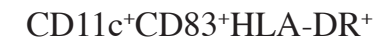 & $147(21.6 \%)$ & $41(3.9 \%)$ & $\mathrm{P}<0.001$ \\
\hline $\mathrm{CD} 16^{+} \mathrm{NCR}^{+}$ & $83(12.2 \%)$ & $41(3.9 \%)$ & $\mathrm{P}<0.001$ \\
\hline $\mathrm{CSF} 1 \mathrm{R}^{+} \mathrm{MSR}^{+}$ & $86(12.7 \%)$ & $69(6.6 \%)$ & $\mathrm{P}<0.001$ \\
\hline \multicolumn{4}{|c|}{ Cytokine and enzyme $(\mathrm{FC}>2)$} \\
\hline IFNG & $481(68.1 \%)$ & $253(22.2 \%)$ & $\mathrm{P}<0.001$ \\
\hline TNFA & $247(34.9 \%)$ & $163(14.3 \%)$ & $\mathrm{P}<0.001$ \\
\hline IL12 & $175(24.8 \%)$ & $200(17.6 \%)$ & $\mathrm{P}<0.001$ \\
\hline VEGFA & $349(49.4 \%)$ & $394(34.7 \%)$ & $\mathrm{P}<0.001$ \\
\hline TGFB1 & $255(36.1 \%)$ & $238(20.9 \%)$ & $\mathrm{P}<0.001$ \\
\hline IL6 & $307(43.4 \%)$ & $300(26.4 \%)$ & $\mathrm{P}<0.001$ \\
\hline IL10 & $112(15.8 \%)$ & $67(5.9 \%)$ & $\mathrm{P}<0.001$ \\
\hline GZMB & $535(76.0 \%)$ & $354(31.4 \%)$ & $\mathrm{P}<0.001$ \\
\hline PRF & $290(41.2 \%)$ & $89(7.9 \%)$ & $\mathrm{P}<0.001$ \\
\hline
\end{tabular}

${ }^{a}$ The SNV number is shown as the mean value and was statistically compared using the Student's t-test. All other parameters were analyzed using Pearson's Chi-square test. ${ }^{\mathrm{b}} \mathrm{Gene}$ amplification: $\mathrm{FC} \geq 5$ and $\mathrm{CN} \geq 6$. A P-value $<0.05$ was considered to indicate a statistically significant difference. TME, tumor microenvironment.

Table II. Gene expression profiling comparing between TME immune types: Hypermutator tumors.

\begin{tabular}{llllr}
\hline Category & Gene Symbol & Probe ID & P-value & Log FC \\
\hline A vs. B & CD19 & A_23_P113572 & $2.04 \times 10^{-5}$ & 2.41 \\
A vs. B & LTB & A_33_P3248265 & $3.55 \times 10^{-8}$ & 2.13 \\
A vs. B & TIGIT & A_33_P3342056 & $1.21 \times 10^{-9}$ & 2.12 \\
A vs. B & CXCR6 & A_23_P109913 & $1.21 \times 10^{-9}$ & 2.12 \\
A vs. B & IDO1 & A_23_P112026 & $3.79 \times 10^{-5}$ & 2.10 \\
A vs. B & BTLA & A_33_P3358923 & $3.65 \times 10^{-5}$ & 2.08 \\
A vs. B & TNFRSF17 & A_23_P37736 & $9.64 \times 10^{-3}$ & 2.08 \\
A vs. B & CD3G & A_23_P98410 & $5.57 \times 10^{-10}$ & 2.01 \\
A vs. C & IL6 & A_23_P71037 & $2.36 \times 10^{-8}$ & 6.71 \\
A vs. C & GZMB & A_23_P117602 & $8.93 \times 10^{-17}$ & 3.98 \\
B vs. C & IL6 & A_23_P71037 & $1.21 \times 10^{-6}$ & 6.41 \\
B vs. C & TREM1 & A_33_P3319905 & $2.16 \times 10^{-9}$ & 3.29 \\
B vs. C & CCR10 & A_33_P3221303 & $4.72 \times 10^{-4}$ & -1.90 \\
\hline
\end{tabular}



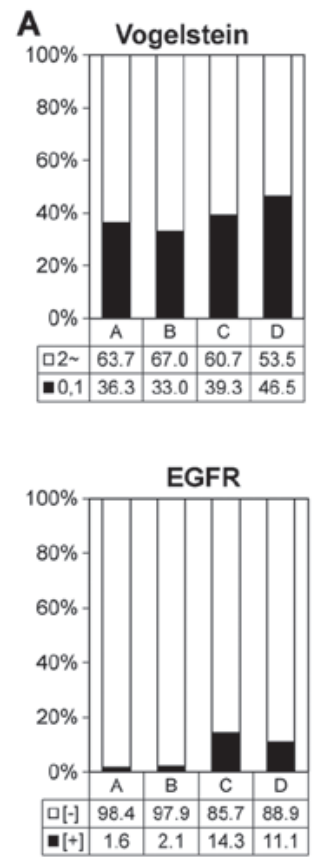

TP53

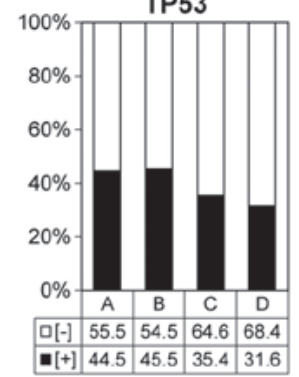

PIK3CA

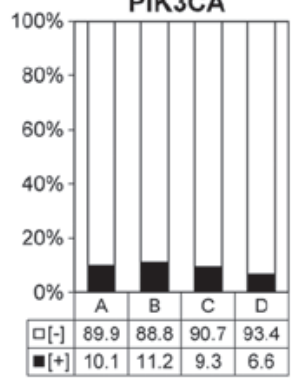

KRAS

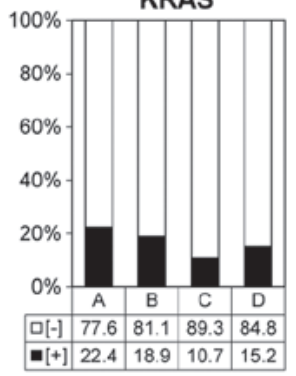

B

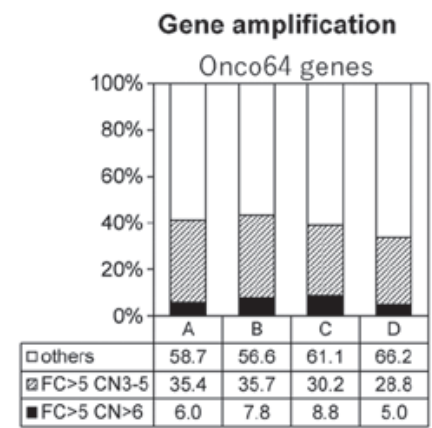

EGFR
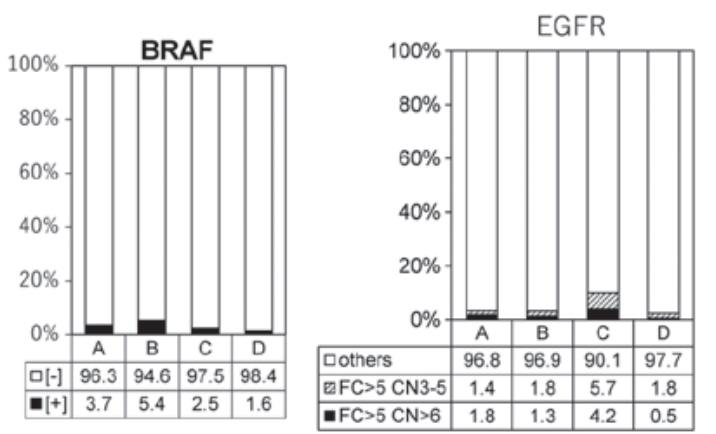

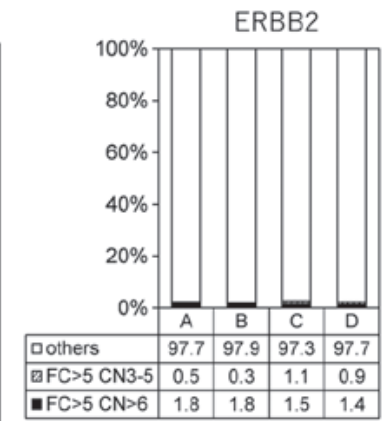

Figure 2. Association of Vogelstein driver gene mutations and gene amplification with TME immune types. (A) The proportions of Vogelstein driver gene (138 genes), TP53, KRAS, EGFR, PIC3CA and BRAF mutations in each immune type. (B) The proportions of all 64 gene amplifications, EGFR gene amplification, and HER2 gene amplification in each immune type. Gene amplification was defined as a $>5$-fold upregulation in expression and $>6$ copy numbers. Each value represents the proportion in percentage of genetic mutations or amplification in each immune type. TME, tumor microenvironment.
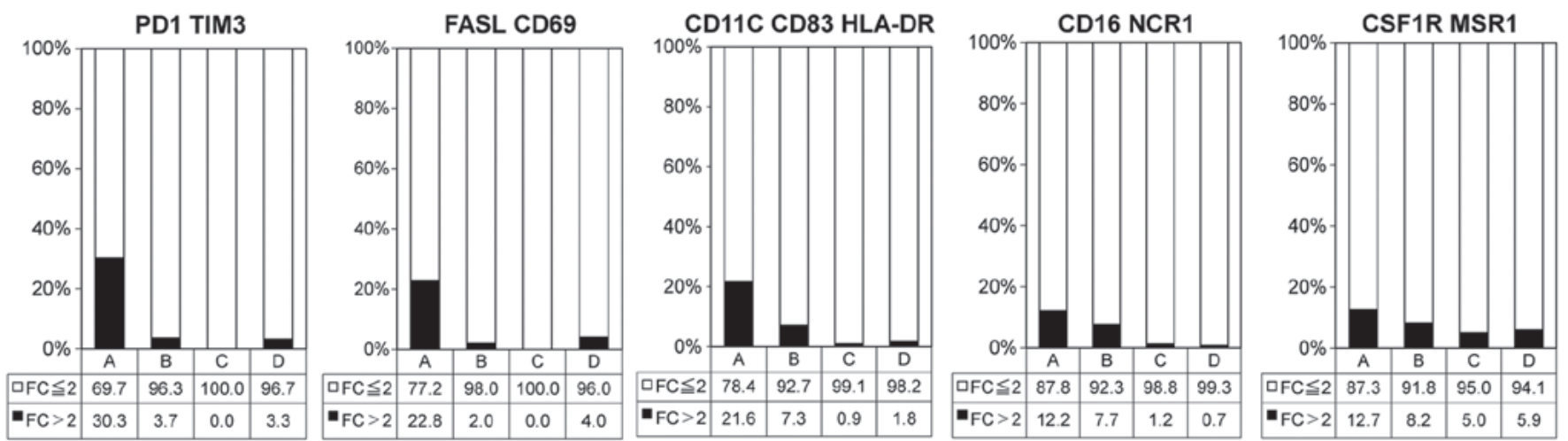

Figure 3. Association of immune cell subpopulations with TME immune types. The frequencies of exhausted T-cell (PD-1 $1^{+}$TIM3 ${ }^{+}$), activated effector T-cell $\left(\right.$ FAS ligand $\left.{ }^{+} \mathrm{CD} 69^{+}\right)$, activated natural killer $(\mathrm{NK})$ cells $\left(\mathrm{CD} 16^{+} \mathrm{NCR} 1^{+}\right)$, mature dendritic cell $\left(\mathrm{CD} 1 \mathrm{c}^{+} \mathrm{CD} 83^{+} \mathrm{HLA}-\mathrm{DR}{ }^{+}\right)$and tumor-associated macrophages $\left(\mathrm{CSFR} 1^{+} \mathrm{CD} 204^{+}\right)$in each TME immune type are shown. Each value represents the proportion in percentage of the immune cell phenotype high $(>2$-fold) and low (<2-fold) expression group in each TME immune type. TME, tumor microenvironment.

of 174 immune response-associated genes, the immune pathway-specific characterization of each TME immune type was performed using IPA software in the radar chart. Immune type A was associated with the Th1 and T-cell activation (PKC, calcium and ICOS) pathway and dendritic cell maturation signaling. On the other hand, type $\mathrm{C}$ exhibited a downregulation in T-cell activation signaling, although the $\mathrm{Cdc} 42$ and OX40 (NF- $\kappa \mathrm{B})$ signaling pathways were activated (Fig. 7A). Moreover, radar chart analysis based on the Vogelstein driver gene expression data revealed that type $\mathrm{C}$ was activated with cancer signaling pathways, such as TGF- $\beta$, ERK/MAPK, NF- $\mathrm{KB}$, EGF, VEGF and JAK/STAT signaling, and was suppressed with T-cell activation signaling (PI3K and $\mathrm{p} 53$ and apoptosis pathways). However, these tendencies were reversed in the type A group (Fig. 7B). In terms of calculated $\mathrm{Z}$ scores, type A exhibited the highest score of the immuno-activation signaling pathway and the lowest score of the cancer pathway signaling.

Hypermutator-related biomarker identification based on $P D-L 1$ and $C D 8 B$ gene expression. The frequency of the SNV number in each TME immune types is shown in Fig. 8A. The frequency of hypermutator tumors (4.5\%, 78 of 1,734 cases) with $>500$ SNVs was significantly higher in types A and B, compared with types $\mathrm{C}$ and D. Hypermutator cases are displayed as black squares in Fig. 8B based on the expression levels of the PD-L1 and CD8B genes. Most hypermutators belonged to immune types A, B and C. Of note, only one case was recognized in immune type $\mathrm{D}\left(\mathrm{PD}-\mathrm{L1}^{+} \mathrm{CD}^{2} \mathrm{~B}^{-}\right)$. Moreover, the expression of 174 immune response-associated genes 

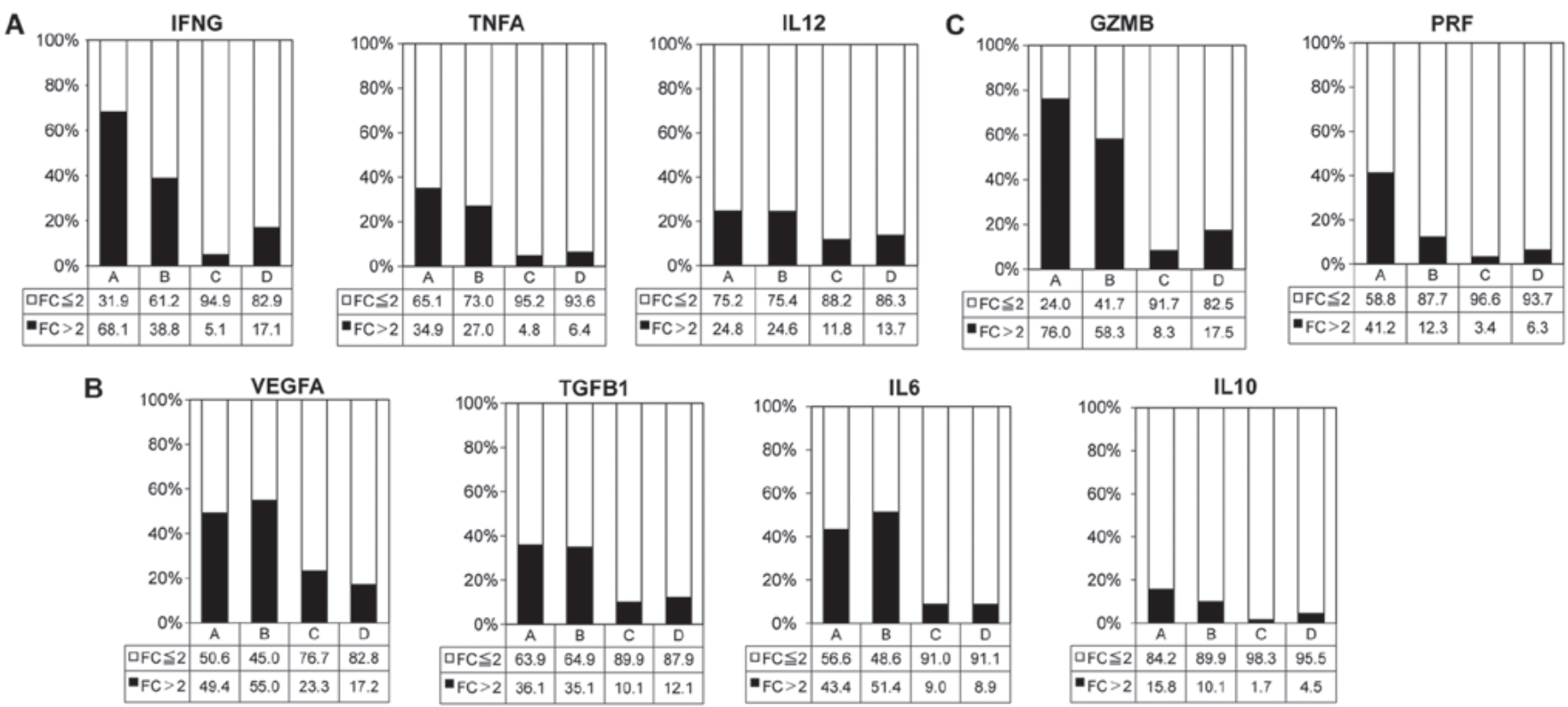

Figure 4. Association of expression in cytolysis and helper T-cell-related cytokine genes with TME immune types. (A) Immuno-activating cytokines. (B) Immuno-suppressive cytokines. (C) Cytolytic factor genes. Each value shows the proportion in percentage of high cytokine gene expression ( $>2$-fold) or low cytokine gene expression (<2-fold) in each TME immune type. TME, tumor microenvironment.

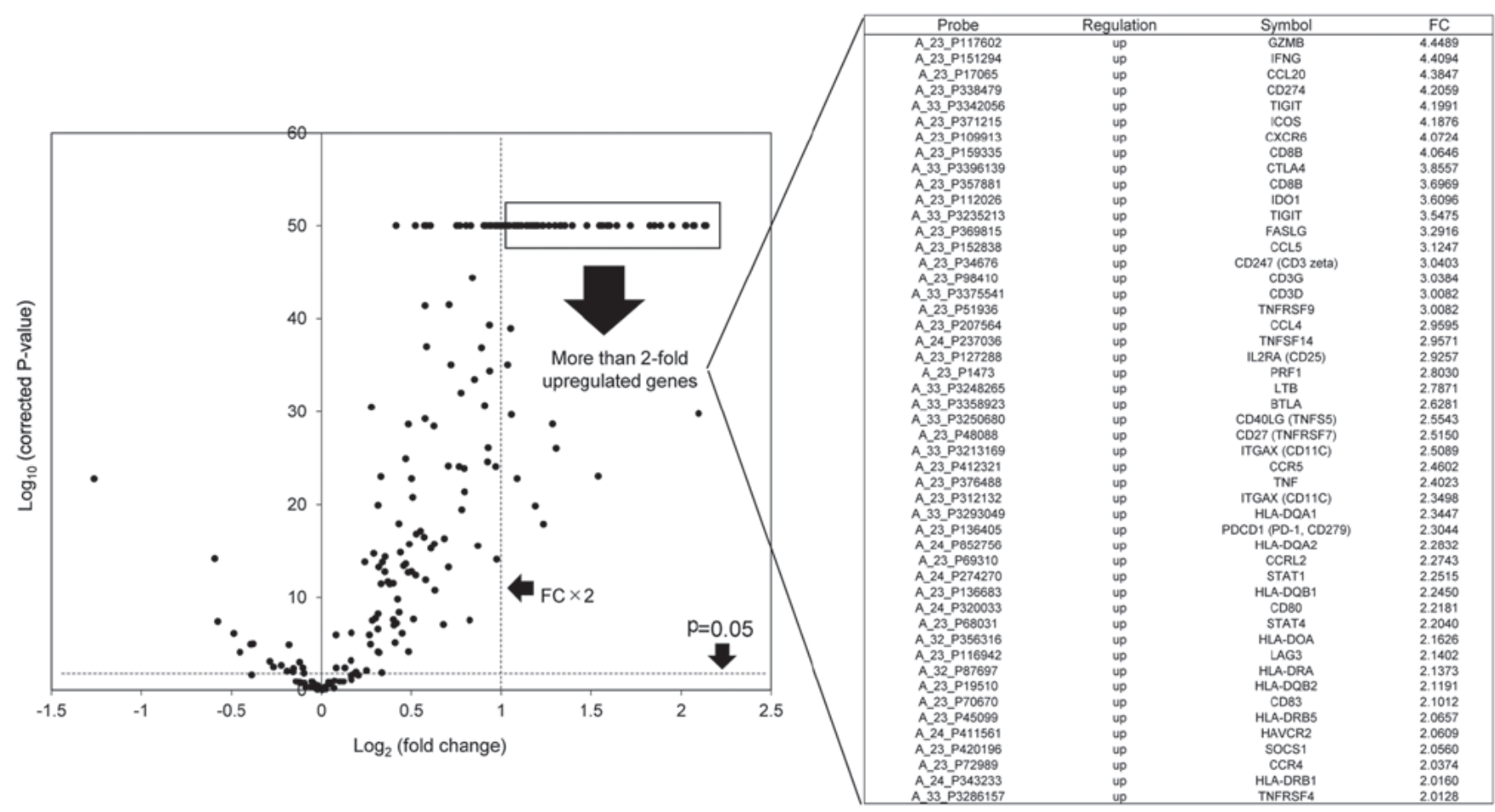

Figure 5. Identification of upregulated immune response-associated genes in TME immune type A compared with other types. A total of 46 upregulated immune response-associated genes with a $>2$-fold change in expression were identified using the volcano plot. The upregulation of all listed genes was statistically significant $(\mathrm{P}<0.05)$. TME, tumor microenvironment.

was compared between hypermutator cases in each immune type using the volcano plot analysis, which indicated that the IL6 gene was upregulated in types A and B compared with type $\mathrm{C}$ (Fig. 8D and E; Table II), and that the TREM1 gene was upregulated in type B compared with type C. Additionally, dominant genes were not recognized when we compared immune response-associated gene expression between type A and type $\mathrm{B}$ (Fig. $8 \mathrm{C}$ and Table II).

\section{Discussion}

Regarding the immune checkpoint blockade for PD-1/PD-L1 in clinical trials, a positive PD-L1 expression, a high mutation burden, a high microsatellite instability (MSI) status and a high TIL status are considered to be possible biomarkers for clinical responder prediction and a good prognosis in a variety of solid tumors treated with PD-1/PD-L1 inhibitors (11-13). 


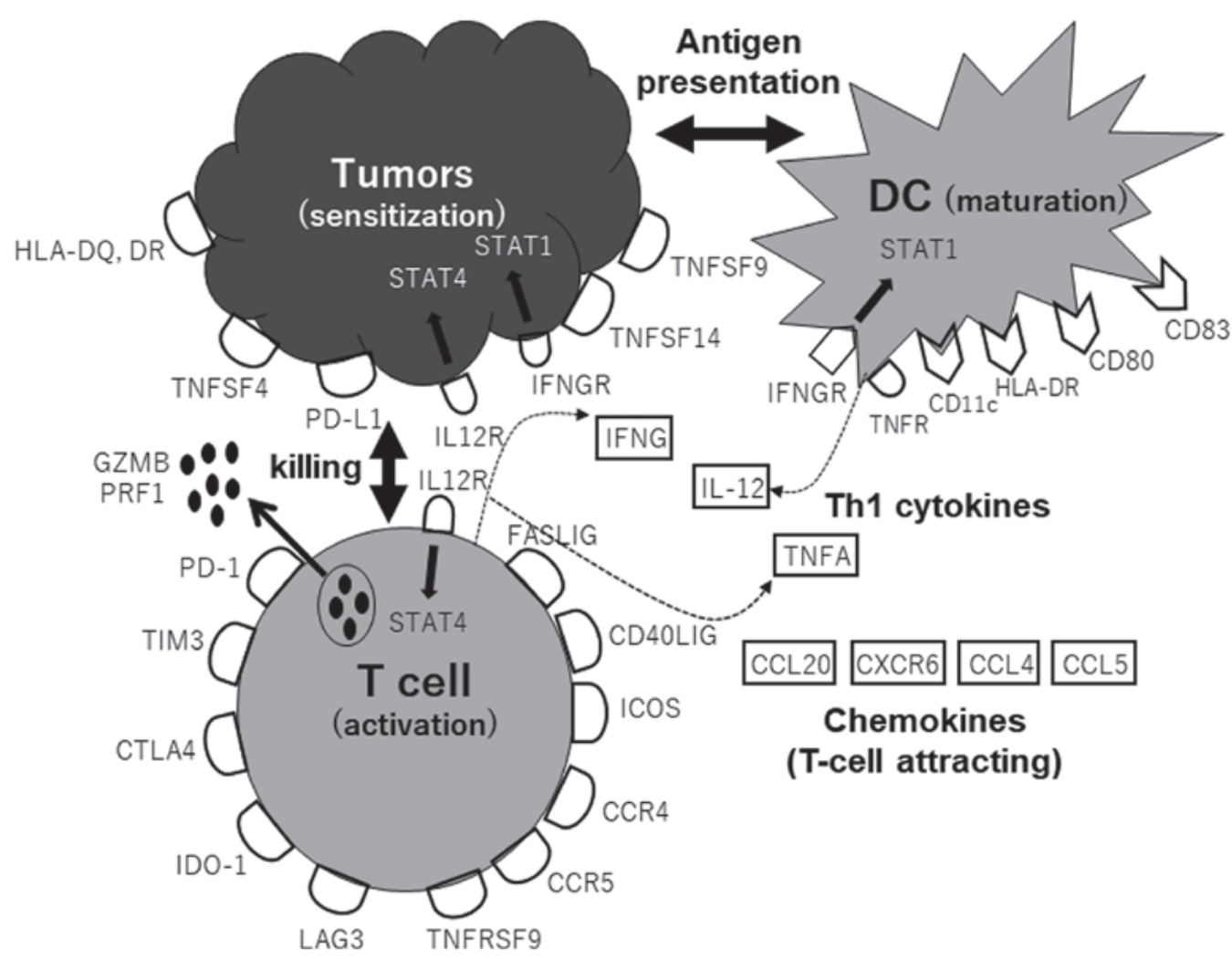

Figure 6. The schema of immune cell network favoring Th1 activation. The upregulated immune response-associated genes identified in Fig. 5 were mainly classified as Th1 pathway-activating gene population, such as T-cell effector activation, CTL killing, T-cell attraction, DC maturation and Th1 cytokines, leading to the durable antitumor effect with the help of sensitization by antigen-presenting cells.
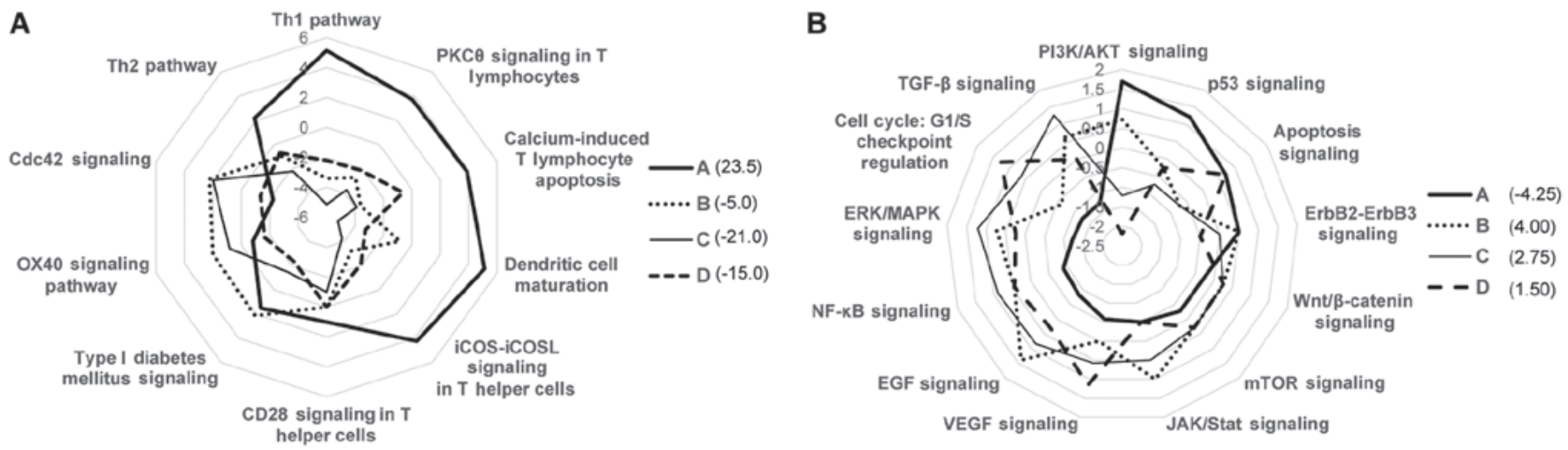

Figure 7. Association of TME immune types with immune or cancer signal pathways. Based on the expression data from the immune response-associated gene or Vogelstein driver gene, immune or cancer signal pathway-specific characterization of each TME immune type was performed using Ingenuity Pathway Analysis software in the radar chart. (A) Immune signal pathway profiling derived from 174 immune-response associated gene expression data. (B) Cancer signal pathway profiling from 138 Vogelstein driver gene expression data in each TME immune type. Each point indicates the Z score showing a tendency of immune or cancer signal pathways. The values in the parenthesis show the sum of $Z$ score in each immune type. TME, tumor microenvironment.

The novelties about the current approach to immunological classifications by PD-L1 and CD8B expression are the following: i) profiling specific immunological marker-positive cell populations including effector and exhausted T-cells, macrophages, dendritic cells and NK cells; ii) immune response-associated gene expression-based immune pathway analysis; and iii) Vogelstein driver gene expression-based cancer signal pathway analysis.

First, genome-based immune cell characterization other than IHC or living-cell methods, such as TILs-based surface marker analysis, is not a widely used method, but few studies on GEP-based immune cell characterization in tumors have been published (21). Thus, there is not much evidence for or against such an approach. However, it is likely that specific immune cell distribution can be recognized with the combination of other gene expression data, such as cytolytic activity, Th1 and Th 2 cytokine production and other immune response-associated genes (22-28).

Second, in this study, the association of TME immune types with immune or cancer signaling pathways was investigated based on GEP data of immune response-associated genes or Vogelstein driver genes. The radar chart demonstrating the 
A

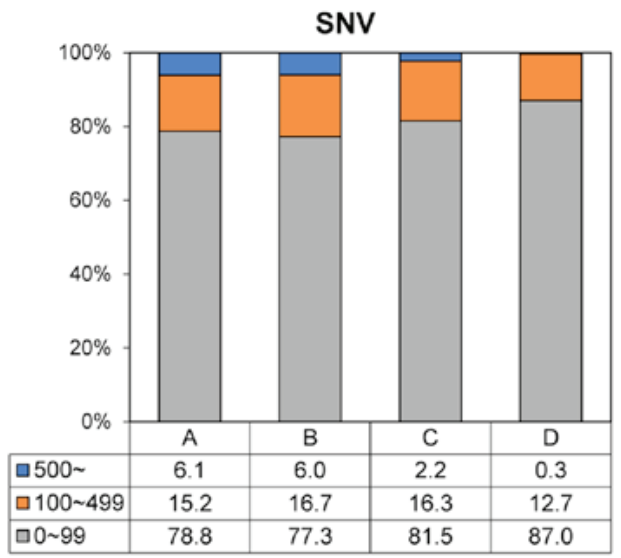

B

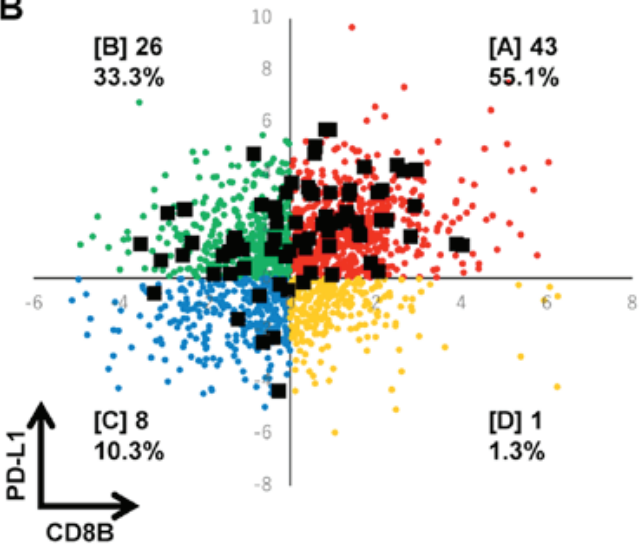

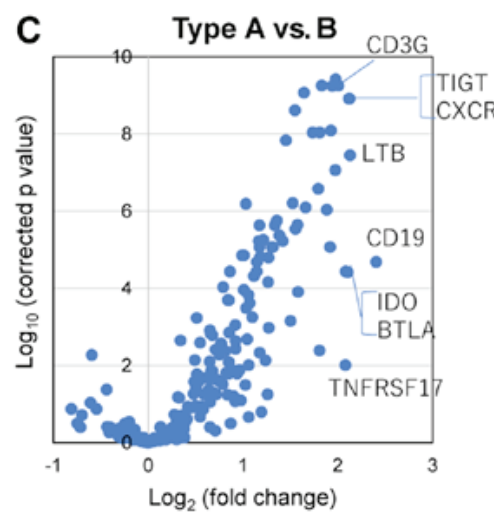
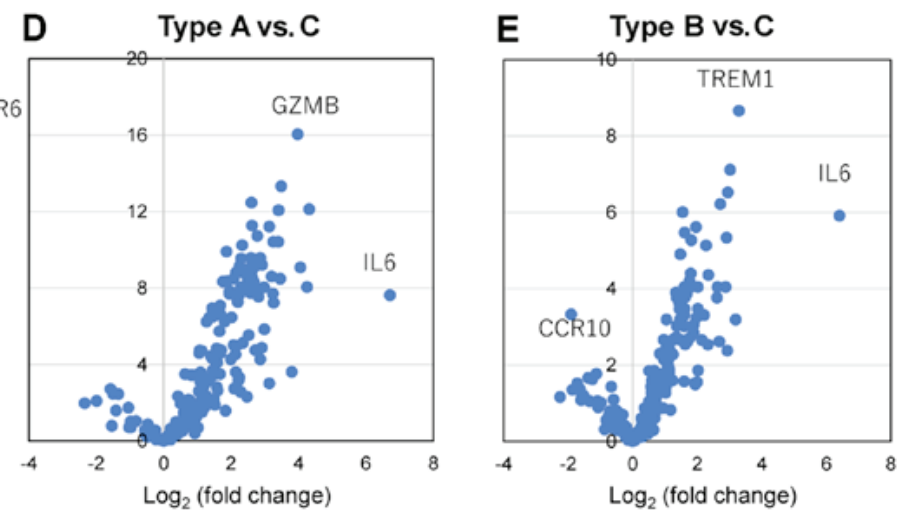

Figure 8. Characterization of hypermutator tumors based on TME immune type-associated gene expression profiling. (A) The proportion of hypermutator tumors with $>500$ SNVs in each TME immune type. (B) The distribution schema of 87 hypermutator tumors in each TME immune type. Types A, B, C and $\mathrm{D}$ are indicated in square brackets. The differentially expressed genes were identified using the volcano plot analysis by the comparison of 174 immuneresponse associated gene expression data between TME immune type-based hypermutators. (C) Type A versus B. (D) Type A versus C. (E) Type B versus C. TME, tumor microenvironment.

association of each immune type with various pathways was helpful to recognize which signal pathways were associated with type A (immune activation) and type C (immune suppression), respectively.

In sum, immune type A was associated with the Th1 T-cell pathway, NK cell activation pathway, dendritic cell maturation and cancer-apoptosis activation signals, and exhibited the highest score in immune-activation signaling pathways. On the other hand, type $\mathrm{C}$ exhibited a downregulation of T-cell activation signaling (with the lowest score) and oncogene signal pathway activation. These results suggested that types $\mathrm{A}$ and $\mathrm{C}$ exhibited opposite characteristics of immune and cancer signal pathways. Our approach is a novel one; thus, the combination of immune-type classification and signaling pathway analysis is a better method to evaluate immunological status prior to specific cancer immunotherapies.

Furthermore, clinical information regarding survival time is suggested to be associated with the current immune type classification. The preliminary observation, demonstrated by Ock et al (15) using survival data from TCGA database, revealed that immune subtype I $\left(\mathrm{PD}-\mathrm{L1}^{+} \mathrm{CD} 8^{+}\right)$exhibited a longer overall survival time compared with subtype III $\left(\mathrm{PD}-\mathrm{L1}^{+} \mathrm{CD} 8^{-}\right)$. We are planning a similar survival analysis in the project HOPE in the near future.

In addition to a high PD-L1 expression and CD8B upregulation as TIL marker, a high load of somatic mutations (hypermutation) in the tumor is another crucial possible biomarker that can predict the good response of immune checkpoint blockade (29-31). In this study, we divided hypermutator tumors with $>500 \mathrm{SNV}$ numbers into the 4 immune types (Fig. 8B). Ock et al demonstrated that the somatic SNV number was significantly higher in immune type A. Of note, our data indicated that hypermutators were found mainly in type A (48\%) and B (35\%), and even some in type $\mathrm{C}\left(16 \%\right.$, $\left.\mathrm{PD}-\mathrm{L1}^{-} \mathrm{CD} 8 \mathrm{~B}\right)$, but not in type $\mathrm{D}$ ( $\left.\mathrm{PD}-\mathrm{L1}^{-} \mathrm{CD} 8 \mathrm{~B}^{+}\right)$. These results suggest that PD-L1 upregulation is a crucial feature for the induction of a durable CTL response against cancers and that hypermutators alone are not sufficient. Additionally, comparing immune response-associated gene expression between TME immune type $\mathrm{A}$ and $\mathrm{C}$ derived from hypermutators revealed the upregulation of specific genes, such as IL6 and GZMB. These observations may indicate that the upregulation of these immunological genes mediated by neoantigen boosting originating from hypermutation can lead to PD-L1 upregulation, resulting in a potent immune checkpoint blockade. Of note, the TREM1 gene was associated with PD-L1 expression in the absence of CD8B expression between types $\mathrm{B}$ and $\mathrm{C}$, which suggests activated macrophage-mediated PD-L1 induction.

Prat $e t$ al analyzed FFPE-derived tumor RNA on the nCounter system using the PanCancer 730-Immune Panel and identified 23 immune-related genes or signatures linked to antitumor-response by immune checkpoint blockade and progression-free survival (32). These 23 immune-related genes 
included PD-1, PD-L1 and other genes associated with CD8 and CD4 T-cell activation, NK cells and IFN- $\gamma$ activation.

This study demonstrated that immune type A was associated with the Th1 T-cell pathway, NK cell activation pathway, DC maturation and cancer-apoptosis activation signaling, which indicated that such results are associated with immune response-activating characteristic, leading to an antitumor effect.

Finally, our approach, based on comprehensive immune response-associated gene expression data from the HOPE project, may be very informative and helpful to the evaluation of immunological status before immunotherapy and the prediction of antitumor response after immunotherapy. In the future, studies should strengthen their evidence levels by adding updated data from a multi-omics approach, specifically immunohistochemistry and clinical survival information.

\section{Acknowledgements}

The authors would like to thank the staff at the Shizuoka Cancer Center Hospital for their clinical support and sample preparation.

\section{Funding}

No funding was received.

\section{Availability of data and materials}

All data generated or analyzed during this study are included in this published article. As the project HOPE is in progress, and not yet completed, sequencing and expression data have not been deposited in a public database. However, the authors declare that all the other data supporting the findings of this study can be available from the corresponding author upon reasonable request.

\section{Authors' contributions}

YA designed the study, drafted the manuscript, and was responsible for completing the study. RK performed a bioinformatics analysis of immune response-associated gene data derived from a large of number cancer patients. AI, CN and HM supported the bioinformatics analysis by organizing and editing data. TN, $\mathrm{KO}$ and $\mathrm{KU}$ performed the storage and organization of sequencing or gene expression data obtained by WES and GEP and responsible for bioinformatics analysis of whole data. TA, KY and MK supported and provided advice as regards the conception and design of the present study. All authors have read and approved the final draft.

\section{Ethics approval and consent to participate}

The Shizuoka Cancer Center launched project HOPE based on the multi-omics analyses including WES and GEP. Ethical approval for the HOPE study was obtained from the Institutional Review Board at the Shizuoka Cancer Center (Authorization no. 25-33). Written informed consent was obtained from all patients enrolled in the study.

\section{Patient consent for publication}

Not applicable.

\section{Competing interests}

The authors declare that they have no competing interests.

\section{References}

1. Weber JS, O'Day S, Urba W, Powderly J, Nichol G, Yellin M, Snively J and Hersh E: Phase I/II study of ipilimumab for patients with metastatic melanoma. J Clin Oncol 26: 5950-5956, 2008.

2. Hodi FS, O'Day SJ, McDermott DF, Weber RW, Sosman JA, Haanen JB, Gonzalez R, Robert C, Schadendorf D, Hassel JC, et al: Improved survival with ipilimumab in patients with metastatic melanoma. N Engl J Med 363: 711-723, 2010.

3. Topalian SL, Hodi FS, Brahmer JR, Gettinger SN, Smith DC, McDermott DF, Powderly JD, Carvajal RD, Sosman JA, Atkins MB, et al: Safety, activity, and immune correlates of anti-PD-1 antibody in cancer. N Engl J Med 366: 2443-2454, 2012.

4. Brahmer JR, Tykodi SS, Chow LQ, Hwu WJ, Topalian SL, Hwu P, Drake CG, Camacho LH, Kauh J, Odunsi K, et al: Safety and activity of anti-PD-L1 antibody in patients with advanced cancer. N Engl J Med 366: 2455-2465, 2012.

5. Wolchok JD, Kluger H, Callahan MK, Postow MA, Rizvi NA, Lesokhin AM, Segal NH, Ariyan CE, Gordon RA, Reed K, et al: Nivolumab plus ipilimumab in advanced melanoma. N Engl J Med 369: 122-133, 2013.

6. Couzin-Frankel J: Breakthrough of the year 2013. Cancer immunotherapy. Science 342: 1432-1433, 2013.

7. Snyder A, Makarov V, Merghoub T, Yuan J, Zaretsky JM, Desrichard A, Walsh LA, Postow MA, Wong P, Ho TS, et al: Genetic basis for clinical response to CTLA-4 blockade in melanoma. N Engl J Med 371: 2189-2199, 2014.

8. Garon EB, Rizvi NA, Hui R, Leighl N, Balmanoukian AS, Eder JP, Patnaik A, Aggarwal C, Gubens M, Horn L, et al; KEYNOTE-001 Investigators: Pembrolizumab for the treatment of non-small-cell lung cancer. N Engl J Med 372: 2018-2028, 2015.

9. Reck M, Rodríguez-Abreu D, Robinson AG, Hui R, Csőszi T, Fülöp A, Gottfried M, Peled N, Tafreshi A, Cuffe S, et al; KEYNOTE-024 Investigators: Pembrolizumab versus chemotherapy for PD-L1-positive non-small-cell lung cancer. N Engl J Med 375: 1823-1833, 2016.

10. Le DT, Durham JN, Smith KN, Wang H, Bartlett BR, Aulakh LK, Lu S, Kemberling H, Wilt C, Luber BS, et al: Mismatch repair deficiency predicts response of solid tumors to PD-1 blockade. Science 357: 409-413, 2017.

11. Dahlin AM,Henriksson ML, Van Guelpen B, Stenling R, Öberg A, Rutegård J and Palmqvist R: Colorectal cancer prognosis depends on T-cell infiltration and molecular characteristics of the tumor. Mod Pathol 24: 671-682, 2011.

12. Llosa NJ, Cruise M, Tam A, Wicks EC, Hechenbleikner EM, Taube JM, Blosser RL, Fan H, Wang H, Luber BS, et al: The vigorous immune microenvironment of microsatellite instable colon cancer is balanced by multiple counter-inhibitory checkpoints. Cancer Discov 5: 43-51, 2015.

13. Schalper KA, Brown J, Carvajal-Hausdorf D, McLaughlin J, Velcheti V, Syrigos KN, Herbst RS and Rimm DL: Objective measurement and clinical significance of TILs in non-small cell lung cancer. J Natl Cancer Inst 107: dju435, 2015.

14. Rooney MS, Shukla SA, Wu CJ, Getz G and Hacohen N: Molecular and genetic properties of tumors associated with local immune cytolytic activity. Cell 160: 48-61, 2015.

15. Ock CY, Keam B, Kim S, Lee JS, Kim M, Kim TM, Jeon YK, Kim DW, Chung DH and Heo DS: Pan-cancer immunogenic perspective on the tumor microenvironment based on PD-L1 and CD8 T-cell infiltration. Clin Cancer Res 22: 2261-2270, 2016.

16. Yamaguchi K, Urakami K, Nagashima T, Shimoda Y, Ohnami S, Ohnami S, Ohshima K, Mochizuki T, Hatakeyama K, Serizawa M, et al: Prevalence of low-penetrant germline TP53 D49H mutation in Japanese cancer patients. Biomed Res 37: 259-264, 2016. 
17. Ohshima K, Hatakeyama K, Nagashima T, Watanabe Y, Kanto K Doi Y, Ide T, Shimoda Y, Tanabe T, Ohnami S, et al: Integrated analysis of gene expression and copy number identified potential cancer driver genes with amplification-dependent overexpression in 1,454 solid tumors. Sci Rep 7: 641, 2017.

18. Akiyama Y, Kondou R, Iizuka A, Ohshima K, Urakami K, Nagashima T, Shimoda Y, Tanabe T, Ohnami S, Ohnami S, et al: Immune response-associated gene analysis of 1,000 cancer patients using whole-exome sequencing and gene expression profiling-Project HOPE. Biomed Res 37: 233-242, 2016.

19. Brazma A, Hingamp P, Quackenbush J, Sherlock G, Spellman P, Stoeckert C, Aach J, Ansorge W, Ball CA, Causton HC, et al: Minimum information about a microarray experiment (MIAME)-toward standards for microarray data. Nat Genet 29: 365-371, 2001.

20. Vogelstein B, Papadopoulos N, Velculescu VE, Zhou S, Diaz LA Jr and Kinzler KW: Cancer genome landscapes. Science 339: 1546-1558, 2013.

21. Papalexi E and Satija R: Single-cell RNA sequencing to explore immune cell heterogeneity. Nat Rev Immunol 18: 35-45, 2018

22. Teng MW, Ngiow SF, Ribas A and Smyth MJ: Classifying cancers based on T-cell infiltration and PD-L1. Cancer Res 75: $2139-2145,2015$

23. Tosolini M, Kirilovsky A, Mlecnik B, Fredriksen T, Mauger S, Bindea G, Berger A, Bruneval P, Fridman WH, Pagès F, et al: Clinical impact of different classes of infiltrating T cytotoxic and helper cells (Th1, th2, treg, th17) in patients with colorectal cancer. Cancer Res 71: 1263-1271, 2011

24. Ascierto RA, Capone M, Urba WJ, Bifuco CB, Botti G, Lugli A Marincola FM, Ciliberto G, Galon J and Fox BA: The additional facet of immunoscore: immunoprofiling as a possible predictive tool for cancer treatment. J Transl Med 11:54, 2913.

25. Lee HJ, Lee JJ, Song IH, Park IA, Kang J, Yu JH, Ahn JH and Gong G: Prognostic and predictive value of NanoString-based immune-related gene signatures in a neoadjuvant setting of triple-negative breast cancer: Relationship to tumor-infiltrating lymphocytes. Breast Cancer Res Treat 151: 619-627, 2015.
26. Gnjatic S, Bronte V, Brunet LR, Butler MO, Disis ML, Galon J, Hakansson LG, Hanks BA, Karanikas V, Khleif SN, et al: Identifying baseline immune-related biomarkers to predict clinical outcome of immunotherapy. J Immunother Cancer 5: 44, 2017.

27. Ayers M, Lunceford J, Nebozhyn M, Murphy E, Loboda A, Kaufman DR, Albright A, Cheng JD, Kang SP, Shankaran V, et al: IFN- $\gamma$-related mRNA profile predicts clinical response to PD-1 blockade. J Clin Invest 127: 2930-2940, 2017.

28. Stroncek DF, Butterfield LH, Cannarile MA, Dhodapkar MV, Greten TF, Grivel JC, Kaufman DR, Kong HH, Korangy F, Lee PP, et al: Systematic evaluation of immune regulation and modulation. J Immunother Cancer 5: 21, 2017.

29. Johnson DB, Frampton GM, Rioth MJ, Yusko E, Xu Y, Guo X, Ennis RC, Fabrizio D, Chalmers ZR, Greenbowe J, et al: Targeted next generation sequencing identifies markers of response to PD-1 blockade. Cancer Immunol Res 4: 959-967, 2016.

30. Dudley JC, Lin MT, Le DT and Eshleman JR: Microsatellite instability as a biomarker for PD-1 blockade. Clin Cancer Res 22: 813-820, 2016.

31. Gong J, Wang C, Lee PP, Chu P and Fakih M: Response to PD-1 blockade in microsatellite stable metastatic colorectal cancer harboring a POLE mutation. J Natl Compr Canc Netw 15: 142-147, 2017.

32. Prat A, Navarro A, Paré L, Reguart N, Galván P, Pascual T, Martínez A, Nuciforo P, Comerma L, Alos L, et al: Immunerelated gene expression profiling after PD-1 blockade in non-small cell lung carcinoma, head and neck squamous cell carcinoma, and melanoma. Cancer Res 77: 3540-3550, 2017. 